CU-PHYSICS/14-2008

\title{
Universal extra dimension: Violation of Kaluza-Klein parity
}

\author{
Biplob Bhattacherjee \\ Department of Physics, University of Calcutta, 92 A.P.C. Road, Kolkata 700009, India \\ E-mail: bbhattacherjee@gmail.com
}

\begin{abstract}
The minimal Universal Extra Dimension (mUED) model respects the Kaluza-Klein (KK) parity $(-1)^{n}$, where $n$ is the KK number. However, it is possible to have interactions located at only one of the two fixed points of the $S_{1} / \mathcal{Z}_{2}$ orbifold. Such asymmetric interactions violate the KK parity. This kills the cold dark matter component of UED but also removes the upper bound on the inverse compactification radius, and thus non-observation of the KK excitations even at the Large Hadron Collider does not necessarily invalidate the model. Apart from the decay of the lightest $n=1 \mathrm{KK}$ excitation, this leads to collider signals which are markedly different from those in the mUED scenario. The phenomenological consequences of such KK-parity violating terms are explored.
\end{abstract}

Keywords: Universal Extra Dimension, KK Parity violation, Large Hadron Collider

\section{Introduction}

The Universal Extra Dimension model (UED) is one of the minimal possible extensions of the Standard Model (SM) with one or more compactified extra dimensions. The minimal model, henceforth called mUED, was proposed by Appelquist, Cheng and Dobrescu [1], and assumes that all SM fields can propagate in one compactified extra dimension $y$. To get chiral fermions at the zero-th level, one needs an $S_{1} / \mathcal{Z}_{2}$ orbifolding, with two fixed points at $y=0$ and $y=\pi R$, where $R$ is the compactification radius. All extra dimensional models are nonrenormalisable, and can at best be treated as an effective theory valid upto a cut-off scale $M_{s}$. Thus, mUED has two free parameters, $R$ and $M_{s}$, with which one can specify the spectrum at any $n$ level. (The masses of the excited scalars also depend on the SM Higgs boson mass $M_{h}$, so strictly speaking, that is also an input parameter.)

One of the interesting feature of the mUED model is the conservation of the KK number. This comes from the fact that all particles can propagate in the extra dimension and so the momentum along the fifth dimension must be conserved. However, presence of two fixed points breaks the translational symmetry along $y$, so the KK number $n$ is no longer conserved. In principle, there may exist some interactions located only at these fixed points. If the interactions are symmetric under the exchange of the fixed points (this is another $\mathcal{Z}_{2}$ symmetry, but not the $\mathcal{Z}_{2}$ of $y \leftrightarrow-y$ ), the conservation of KK number breaks down to the conservation of KK parity, defined as $(-1)^{n}$, where $n$ is the KK number 2. The mUED assumes the conservation of KK parity. KK parity does not allow single production of $n=1$ paricles and guarantees the stability of the lowest lying $n=1 \mathrm{KK}$ state (LKP). The LKP, for most of the parameter space, is an excitation of the hypercharge gauge boson $B$ and is an excellent cold dark matter (CDM) candidate 3. The mass of LKP is approximately $1 / R$ and hence the overclosure of the universe puts an upper bound on $R^{-1} \leq 800 \mathrm{GeV}$ [4. This guarantees the production of at least the $n=1$ excited states at the Large Hadron Collider (LHC).

While the spectrum for any $n$ is highly degenerate at the tree-level, the radiative corrections lift the degeneracy and provide interesting collider phenomenology [2, 5. There are two types of corrections: the first one, which results just from the compactification of the extra dimension, called bulk correction, is in general small (zero for fermions) and is constant for all $n$ levels. The second one, called boundary correction, is comparatively large 
(goes as $\ln \left(\Lambda^{2} / q^{2}\right)$ ), and plays the major role in determining the exact spectrum and possible decay modes. In the mUED model, it is assumed that the boundary corrections vanish at $q=M_{s}$, and so one can identify the cut-off scale $M_{s}$ with the regularization scale $\Lambda$.

The collider phenomenology of minimal UED has been investigated in detail [6, 7, 8, 9, A crucial feature of these studies is the existence of the LKP and hence the missing energy and missing transverse momentum signal at the colliders, which stems from the conservation of KK parity. However, it is possible to have fixed-point located interactions that are asymmetric in nature [1. This violates the KK parity, analogous to the R parity violation in supersymmetry. Some phenomenology of such KK parity violation were also discussed in [8, 10]. The possibility of KK parity violation with a 'partial' universality (by not allowing, or only partially allowing, the Higgs boson to flow in the fifth dimension) has also been discussed by [11. KK-parity violation, in the context of other extra dimensional models, has also been considered in [12].

The aim of this paper is to investigate the phenomenology of such a KK parity violating (KKPV) model where a fixed-point located asymmetric term is responsible for KKPV. Unless the KKPV couplings are uninterestingly tiny, the LKP will decay within the lifetime of the universe and will not be a CDM candidate anymore. While this removes one of the main motivations of UED, this also enlarges the parameter space by removing the upper bound coming from the CDM density. In other words, we plan to answer this question: If LHC does not find a KK excitation within the overclosure bound, which, taking into the possibility of a heavy SM Higgs boson and the subsequent relaxation of the said bound, is about $1.4 \mathrm{TeV}$ [13], does it mean that the UED model is ruled out? We will show that the answer is negative, and point out the major signals coming from such a model.

A major spin-off of the model is the possibility to accommodate graviton states. In the conventional UED model with conservation of KK parity, gravitons cannot be accommodated for $R^{-1} \leq 800 \mathrm{GeV}[3$ as the model would allow an unacceptably large rate of $\gamma_{1} \rightarrow \gamma+G_{1}$ (where $G_{1}$ is the first excited graviton state) which is ruled out from the cosmological diffuse photon flux. However, if KK parity is violated, the excited photons would decay to conventional fermion-antifermion pairs and not to gravitons, as the latter process is suppressed by the Planck mass. Thus, there are no dangerous gravitons in the model.

The plan of this article is as follows. We first describe some of the basic features of UED model required for our analysis. Readers familiar with the formalism of UED can directly go to Section 3, where the KK parity violation is introduced. In Section 4, the nature and various decay modes of the erstwhile stable LKP, and also the NLKP (next-to-lightest KK particle) are discussed. We show the various possible combinations of LKP and NLKP depending on the model parameters. In Section 5, we briefly touch upon the collider signatures of such modes at the LHC. Finally Section 6 summarizes the results and addresses the possible issues of this work.

\section{The mUED model}

The model has been discussed in great detail in the literature. In this section we briefly mention some of the interesting features of UED required for our analysis.

- The tree level mass spectrum for any level $n$ is almost degenerate. The masses of these KK modes are given (at tree level) by $M_{n}^{2}=M_{0}^{2}+\left(n R^{-1}\right)^{2}$, where $M_{0}$ is the mass of the corresponding SM particle. The tree level relation is modified when radiative corrections are taken into account 2, 14, 15. This causes significant splitting among the particle masses of any KK level and has important effects on collider phenomenology. The one loop corrected masses are determined by $R^{-1}$ and $\Lambda$, the cut off scale. In order to determine the excited scalar masses the SM Higgs mass $\left(M_{h}\right)$ is also required.

- All $n=1$ particles have to be pair produced and ultimately they must cascade down to the lowest lying $n=1$ particle (LKP), due to the conservation of KK parity. However, as the mass splitting among the $n=1$ states is generally small (being induced by radiative corrections), the final state will be soft leptons or jets associated with missing transverse momentum. While the missing energy is large, the missing $p_{T}$ is small, because the spectrum is still quasi-degenerate. 
- For most of the parameter space $\left(R^{-1} \leq 800 \mathrm{GeV}\right)$ the $n=1$ photon, which is almost the excitation of the hypercharge gauge boson $B$ due to the smallness of the 'Weinberg angle' for the $n>0$ levels, is the LKP. (If gravitons are included, they become the LKP and the model runs into trouble with the diffuse photon flux coming from $B_{1} \rightarrow \gamma+G_{1}$, so we exclude gravitons). For higher values of $R^{-1}$ and for high $M_{h}, H_{1}^{ \pm}$, the charged Higgs boson excitation, becomes the LKP. This possibility cannot be encouraged if LKP is stable and a prospective CDM candidate.

- The corrections for KK modes with electroweak interactions are generally small. Singlet or doublet leptons lie just above the $B_{1}$. Masses of the three excited scalars $h_{1}, A_{1}^{0}$, and $H_{1}^{ \pm}$are also very close to the excited lepton masses (see, e.g., 2] for a benchmark spectrum). However, the scalar masses depend on the SM Higgs mass $M_{h}$; if we keep $R^{-1}$ and $\Lambda$ fixed, for larger $M_{h}, H_{1}^{ \pm}$and $A_{1}^{0}$ masses go down and $h_{1}$ becomes more massive. The charged scalar mass can even go below the $B_{1}$ mass $[3]$.

- The mass equation for the excited scalars may contain a universal boundary-located soft term $\overline{m_{h}^{2}} 2$. This term is a free parameter of the theory and is taken to be zero in the mUED model. For fixed $M_{h}$, all excited scalar masses increase with increasing $\overline{m_{h}^{2}}$. However, large negative values of $\overline{m_{h}^{2}}$ again drives

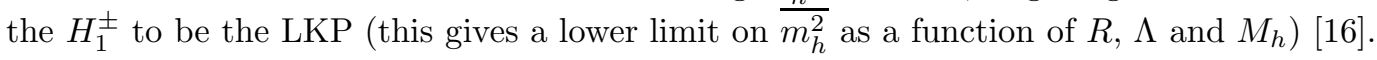

- The $n=1$ fermions are vectorial and can be $\mathcal{Z}_{2}$-even (left doublet and right singlet) or $\mathcal{Z}_{2}$-odd (left singlet and right doublet). These states are not exactly the mass eigenstates. In the doublet-singlet basis, we get a non-diagonal mass matrix, whose off-diagonal entries are the zeroth level mass $M_{0}$. So the mass matrix is almost diagonal for all fermions, except for the third generation quarks, in particular for the top. After diagonalisation and a chiral rotation, one gets the proper mass eigenstates.

\section{KK parity violation}

In this section we study the effect of localised kinetic operators on the boundary. The possibility of such terms has been mentioned in [1, 8, 10. Let us consider, as an illustrative example, the simplest possible one, i.e., the fermion kinetic term, which, located at $y=y_{0}$, looks like

$$
L_{f}=\frac{\lambda}{2 M_{s}} \int\left[i \bar{\psi} \Gamma^{\alpha} D_{\alpha} \psi-i\left(D_{\alpha}^{\dagger} \bar{\psi}\right) \Gamma^{\alpha} \psi\right] \delta\left(y-y_{0}\right) d y,
$$

where $\psi\left(x^{\mu}, y\right)$ is any five dimensional fermionic field and $\lambda$ is the coupling constant. The term is suppressed by the cutoff scale $M_{s}$, which may be identified with $\Lambda$. Such a term contributes to the kinetic term of the KK fermion and hence changes its mass. To determine the spectrum, we have made several simplifying assumptions, without seriously compromising with the phenomenology.

- The first assumption is to place such a term only at $y=0$ without losing any generality as the points $y=0$ and $y=\pi R$ have already been chosen as the fixed points for the orbifold and the decomposition into even and odd modes are performed accordingly. For this case, only even modes will mix with each other.

- To concentrate on the LHC-related phenomenology, let us consider the mixing between $n=0$ and $n=1$ states only. In principle, all $n$ states can mix with each other, but the admixture of the higher states in the low-lying physical states are suppressed by their masses.

- We take the KK parity violating effects, parametrised by the dimensionless coupling

$$
h=\frac{\lambda}{2 \pi M_{s} R},
$$

to be small (it should be perturbative for any meaningful calculation). In fact, we would take $h$ to be so small, $\mathcal{O}\left(10^{-2}\right)$, that effects on the spectrum that depends quadratically on $h$ can be neglected. In this limit, it is enough to compute the tree-level corrections to the spectrum and neglect the loop effects. 
- We take $\lambda$ and hence $h$ to be the same for all fermionic flavours. This is in conformity with lepton universality and suppression of tree-level FCNCs. On the other hand, non-uniformity of $\lambda$ may be constrained from such low-energy observables, in analogy with the R-parity violating couplings of supersymmetry.

- If $h$ is small, we can take the standard KK expansion of the fields as defined in 11. For large values of $h$, the expansion is most definitely not valid, but if we neglect terms $\mathcal{O}\left(h^{2}\right)$ and higher, one can use the standard perturbation theory with the $h=0$ limit as the unperturbed basis.

We now integrate over $y$ and get the usual 4-d Lagrangian. The kinetic part of the 4-d Lagrangian in the $(n=0$, $n=1$ doublet, $n=1$ singlet) basis is given by

$$
\frac{i}{2}\left(\begin{array}{ccc}
\psi_{L}^{(0)} & \psi_{L}^{\overline{(1)}} & S_{L}^{(1)}
\end{array}\right) K_{L} i \gamma^{\mu} \partial_{\mu}\left(\begin{array}{c}
\psi_{L}^{(0)} \\
\psi_{L}^{(1)} \\
S_{L}^{(1)}
\end{array}\right)+\frac{i}{2}\left(\begin{array}{lll}
\psi_{R}^{(0)} & \psi_{R}^{\overline{(1)}} & S_{R}^{\overline{(1)}}
\end{array}\right) K_{R} i \gamma^{\mu} \partial_{\mu}\left(\begin{array}{c}
\psi_{R}^{(0)} \\
\psi_{R}^{(1)} \\
S_{R}^{(1)}
\end{array}\right)+h . c,
$$

where

$$
K_{L}=\left(\begin{array}{ccc}
1 / 2+h & \sqrt{2} h & 0 \\
\sqrt{2} h & 1 / 2+2 h & 0 \\
0 & 0 & 1 / 2
\end{array}\right) ; \quad K_{R}=\left(\begin{array}{ccc}
1 / 2+h & 0 & \sqrt{2} h \\
0 & 1 / 2 & 0 \\
\sqrt{2} h & 0 & 1 / 2+2 h
\end{array}\right)
$$

Note that the odd fields $S_{L}^{(1)}$ and $\psi_{R}^{(1)}$ do not mix with the $n=0$ fields. Here $K_{L}$ and $K_{R}$ are two symmetric matrices and they are diagonalised by two orthogonal matrices $E_{L}$ and $E_{R}$. The eigenvalues of $E_{L}$ and $E_{R}$ are $1 / 2,1 / 2$ and $(1+6 h) / 2$. We now rescale the kinetic terms by two normalisation matrices $N_{L}$ and $N_{R}$. After diagonalisation and rescaling, the kinetic terms take their canonical forms. In this intermediate basis, the kinetic terms are diagonal but the mass matrix is not; rather, it is of the form

$$
M^{\prime}=N_{L}^{-1} E_{L}^{T} M E_{R} N_{R}^{-1},
$$

where $M$ is the mass matrix in the KK basis:

$$
M=\left(\begin{array}{ccc}
M_{0} & h \sqrt{2} / R & 0 \\
0 & 2 h / R+1 / R+\Delta_{D} & M_{0} \\
h \sqrt{2} / R & M_{0} & -2 h / R-1 / R-\Delta_{S}
\end{array}\right)
$$

where $M_{0}$ is the $n=0$, i.e., SM fermion mass, and $\Delta_{D}$ and $\Delta_{S}$ are the radiative corrections on $n=1$ doublet and singlet fermions respectively. Their expressions can be found in 2 and do not change in the limit of small $h . M^{\prime}$ is neither diagonal nor symmetric but can be diagonalised by a bi-unitary transformation of the form $M_{D}=V^{\dagger} M^{\prime} U$. The unitary matrices $U$ and $V$ can be obtained by diagonalising $M^{\prime} M^{\prime \dagger}$ and $M^{\prime} \dagger M^{\prime}$ respectively. We can choose $U$ and $V$ in such a way that all elements of $M^{\prime}$ are positive.

The transformation equations which connect original KK basis to the mass basis are given below

$$
\left(\begin{array}{c}
\psi_{L}^{(0)} \\
\psi_{L}^{(1)} \\
S_{L}^{(1)}
\end{array}\right)=N_{R}^{-1} U\left(\begin{array}{c}
\phi_{L}^{(1)} \\
\phi_{L}^{(2)} \\
\phi_{L}^{(3)}
\end{array}\right) ; \quad\left(\begin{array}{c}
\psi_{R}^{(0)} \\
\psi_{R}^{(1)} \\
S_{R}^{(1)}
\end{array}\right)=N_{L}^{-1} V\left(\begin{array}{c}
\phi_{R}^{(1)} \\
\phi_{R}^{(2)} \\
\phi_{R}^{(3)}
\end{array}\right)
$$

The transformation is not unitary in nature as the normalisation matrices are themselves non-unitary.

Once we obtain the physical states, the Feynman rules can be computed from the Lagrangian and the mixing matrix. However, for small $h$ so that $\mathcal{O}\left(h^{2}\right)$ terms can be neglected, the rules are particularly simple, e.g.,

$$
\begin{aligned}
& \overline{e_{1}^{(1)}} e^{(0)} \gamma^{(0)} \Longrightarrow i e h \sqrt{2} \gamma_{\mu}\left(1+\gamma_{5}\right) \text {, } \\
& \overline{e_{2}^{(1)}} e^{(0)} \gamma^{(0)} \Longrightarrow i e h \sqrt{2} \gamma_{\mu}\left(1-\gamma_{5}\right) \text {, } \\
& \overline{e_{1}^{(1)}} e^{(0)} Z^{(0)} \Longrightarrow-\frac{i e h s_{W} \sqrt{2}}{c_{W}} \gamma_{\mu}\left(1+\gamma_{5}\right) \text {, }
\end{aligned}
$$




$$
\begin{aligned}
\overline{e_{2}^{(1)}} e^{(0)} Z^{(0)} & \Longrightarrow \frac{i e h \sqrt{2}}{2 s_{W} c_{W}}\left(1-2 s_{W}^{2}\right) \gamma_{\mu}\left(1-\gamma_{5}\right), \\
\overline{e_{2}^{(1)}} \nu^{(0)} W^{(0)} & \Longrightarrow-\frac{i e h}{s_{W}} \gamma_{\mu}\left(1-\gamma_{5}\right), \\
\overline{e^{(0)}} e^{(0)} B^{(1)} & \Longrightarrow \frac{i e h \sqrt{2}}{2 c_{W}}\left[\gamma_{\mu}\left(1-\gamma_{5}\right)+2 \gamma_{\mu}\left(1+\gamma_{5}\right)\right],
\end{aligned}
$$

where the subscripts refer to the dominant admixture at the $n=1$ level, and the superscripts to the KK numbers themselves. We have not shown the KKPV contributions to the KK-number conserving vertices (e.g., an $n=0$ gauge boson coupling to two $n=1$ fermions), as the standard gauge coupling is overwhelmingly dominant. By the same argument, KKPV decays of an $n=1 \mathrm{~W}, Z$, or gluon to two $n=0$ fermions have not been shown.

\section{III.1 Spectrum}

With $\lambda \sim 1, M_{s} R \sim 10, h_{\max } \sim 0.02$. We study the spectrum by varying $h$ between -0.02 and 0.02 . Note that while this range does not depend on the precise value of $R^{-1}$, the low-energy constraints should depend upon $h R$ and hence with large values of $R^{-1}$, most of these constraints could be successfully avoided. For such small values of $h$, the branching fractions of KK allowed channels will be hardly affected (except for a few cases that we will show later), since they are mostly driven by gauge or large Yukawa couplings. However, the LKP will decay, and decay promptly within the detector unless $h$ is very tiny.

The mixing angles between the KK basis and the mass basis depend upon $R^{-1}$ and $h$, and also indirectly on $\Lambda$ through $\Delta_{D}$ and $\Delta_{S}$. They also explicitly depend on $M_{0}$, numerically important only for the top quark and the otherwise closely spaced levels.

The spectrum for $R^{-1}=500 \mathrm{GeV}$ is shown in fig. 1 The horizontal lines, from top to bottom, stand for excited gluon $g_{1}, W_{1} / Z_{1}$ (they are almost degenerate), and $B_{1}$ respectvely. As expected, the excited gauge boson masses are not affected. There are two quark states for each flavour; they are the linear combinations of the singlet and the doublet fields. We shall call them $q_{1}$ (dominantly singlet) and $q_{2}$ (dominantly doublet). For all fermions, the subscript refers to the SU(2) gauge quantum number, not the KK number, but this should not create any confusion as we are interested in the phenomenology of only the $n=1$ level. The falling lines, from top to bottom, correspond to $u_{2} / d_{2}, u_{1}, d_{1}$ quarks and $l_{2} / \nu_{2}, l_{1}$ leptons respectively. Note that the fermion masses increase for negative $h$ and decrease for positive $h$. For the quarks, the change is about $4 \%$, but there is no level crossing, and hence the decay patterns remain identical. (The channel $q_{1} \rightarrow q_{0} V_{0}$ or $g_{1} \rightarrow q_{0} q_{0}$ opens up, where $V_{0}$ is a SM gauge boson, but the coupling is suppressed and the branching ratios are only minutely modified.) Interesting thing happens for leptons, as their masses are close to the $B_{1}$ mass, and level crossing may take place. For example, for $R^{-1}=500 \mathrm{GeV}$ and $h \approx 0.01$, the dominantly singlet lepton $l_{1}$ can become the LKP and its mUED decay channel to $n=0$ lepton and $B_{1}$ closes. The only possible channel for $l_{1}$ to decay is the KK parity violating one, to a lepton and an $n=0$ electroweak gauge boson. Similarly, for $h<0$ the leptons may go above the $n=1$ scalars (whose masses do not depend on $h$ ) and the scalar decay channels undergo a fundamental change, from two-body $\tau$ modes to three-body $f \bar{f} B_{1}$.

\section{LKP and NLKP: Phase diagram and decay patterns}

If the LKP is no longer the dark matter candidate, it need not be a neutral one any more. Also, depending on the parameters of the model, namely, $R^{-1}, M_{s}=\Lambda, M_{h}$, and $h$, there are various possible NLKPs (we take, for simplicity, $\overline{m_{h}^{2}}=0$ ). The possibilities include $l_{1}$ and $l_{2} B_{1}, H_{1}^{ \pm}$, and $A_{1}^{0}$ (the neutral CP-odd $n=1$ scalar).

The LKP-NLKP phase diagrams are shown in fig. 2, drawn for $M_{s} R=20$, for two distinct cases: a light SM Higgs boson $\left(M_{h}=120 \mathrm{GeV}\right)$ and a heavy SM Higgs boson $\left(M_{h}=250 \mathrm{GeV}\right)$. The salient features are as follows: 


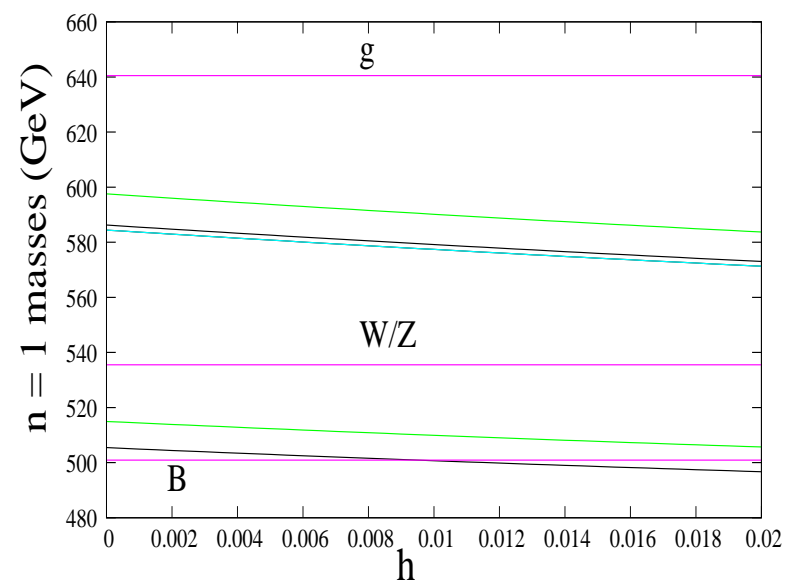

Figure 1: Spectrum for the $n=1$ level. From top to bottom, the sloping lines are for $u_{2} / d_{2}, u_{1}, d_{1}, l_{2} / \nu_{2}$, and $l_{1}$ respectively. For the notation, see text.

Case $1\left(M_{h}=120 \mathrm{GeV}\right)$ : In this case there are only two possible LKP candidates: $B_{1}$ (regions 1 and 3$)$ and $l_{1}$ (region 2). While the $h=0$ limit corresponds to the mUED ( $l_{1}$ NLKP, region 1 ), the transition to $l_{1}$ LKP can be understood from fig. 1 For sufficiently negative values of $h, l_{1}$ goes above $H_{1}^{ \pm}$, which then becomes NLKP (region 3). The parameter space does not allow $H^{ \pm}$as LKP.

Case $2\left(M_{h}=250 \mathrm{GeV}\right)$ : For large $M_{h}$, the situation becomes more complicated. The large quartic selfcoupling drives the $H_{1}^{ \pm}$mass down and in region 2, this becomes the LKP, while it is the usual $B_{1}$ LKP phase in region 1. In region 1 , depending on the values of the model parameters, either $l_{1}$ or $H_{1}^{ \pm}$can be the NLKP, while in region 2 , either $B_{1}, A_{1}^{0}$, or $l_{1}$ is the NLKP. Region 3 is the $l_{1}$ LKP region, with $B_{1}$ or $H_{1}^{ \pm}$as the NLKP. The phase diagram can be more complicated with excited gravitons or right-handed neutrinos, which we have not considered here.
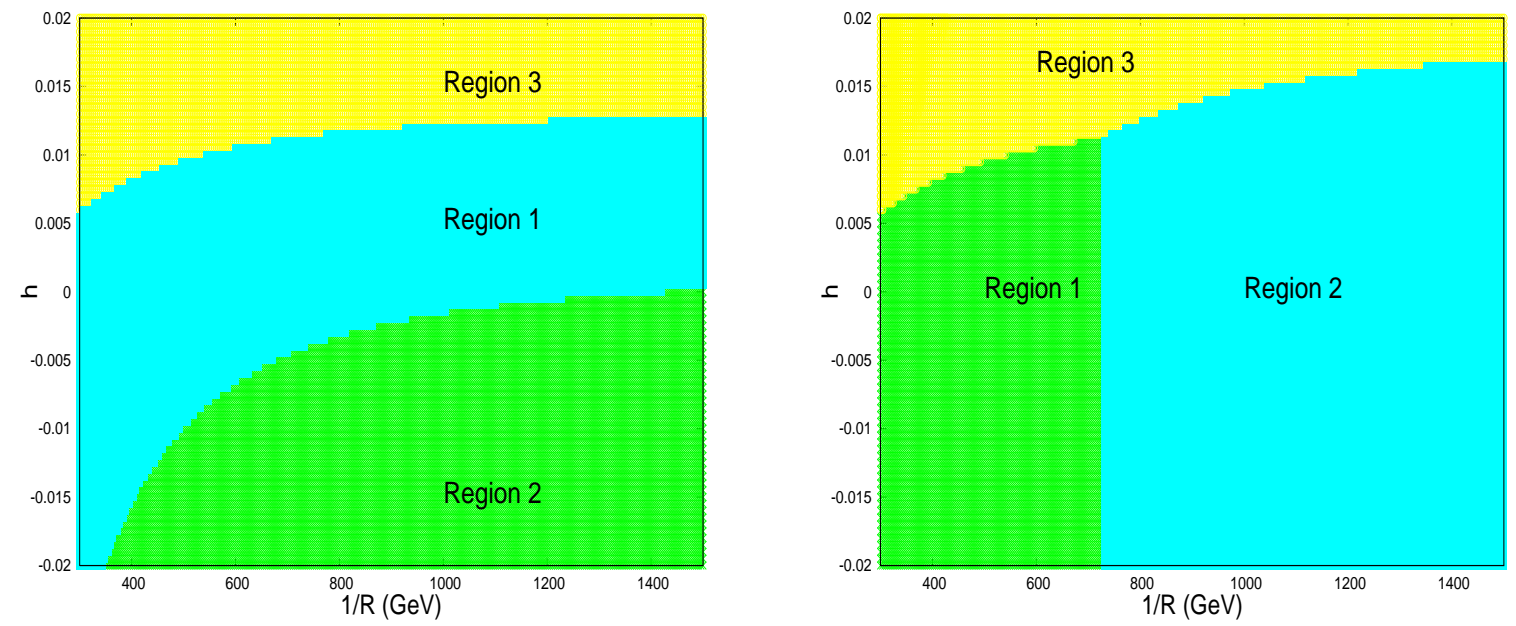

Figure 2: LKP-NLKP phase diagram for $M_{h}=120 \mathrm{GeV}$ (left panel) and $M_{h}=250 \mathrm{GeV}$ (right panel). For the explanation of the various regions, see text. 


\section{IV.1 Decay of LKP and NLKP}

As was mentioned above, there are three possibilities for the LKP: $B_{1}, H_{1}^{ \pm}$and $l_{1}$, depending on the parameter space. The KK parity violating interactions allow couplings of $n=1$ states with two SM particles. There is obviously no KK conserving decay modes for LKP.

Case $1\left(B_{1} \mathrm{LKP}\right): B_{1}$ can decay to two SM fermions, $q \bar{q}, l \bar{l}$ or $\nu \bar{\nu}$. The couplings are proportional to the corresponding hypercharges; the quark channels are also enhanced by the colour factor. The phase space suppression is minimal unless $B_{1}$ is just above the $t \bar{t}$ threshold. The table shows the $B_{1}$ branching fractions for $R^{-1}=500 \mathrm{GeV}$ (there is no KK conserving decay, so the widths do not depend on the value of $h$ ).

\begin{tabular}{||c|c|c||}
\hline $\begin{array}{c}\operatorname{Br}\left(B_{1} \rightarrow q \bar{q}\right) \\
(\%)\end{array}$ & $\begin{array}{c}\operatorname{Br}\left(B_{1} \rightarrow e^{ \pm}, \mu^{ \pm}, \tau^{ \pm}\right) \\
(\%)\end{array}$ & $\begin{array}{c}\operatorname{Br}\left(B_{1} \rightarrow \nu_{e}, \nu_{\mu}, \nu_{\tau}\right) \\
(\%)\end{array}$ \\
\hline 53.3 & 38.9 & 7.8 \\
\hline
\end{tabular}

Table 1: Branching fractions of $B_{1}$.

The hadron channels are difficult to identify at the LHC, except maybe the $t \bar{t}$ channel. A better option is to look for the dilepton channel whose invariant mass peaks at $M_{B_{1}}$. If $B_{1}$ is the NLKP then it almost always decays to $B_{1} \rightarrow l_{1} l_{0}$, followed by the KK parity violating decays of $l_{1}$ to $l$ plus $\gamma$ or $Z$ (the $l_{0}$, being mostly right-chiral as it is produced in a vector interaction in association with $l_{1}$, has a very small branching ratio to $\nu+W)$.

There is an interesting possibility. If $h$ is sufficiently small, lifetime of $B_{1}$ may be long enough, so that it can decay outside the detector. Such situation can mimic the standard mUED scenario where LKP is stable. One has to calculate the lifetime of $B_{1}$ as function of $h$ for different values of $R^{-1}$. In figure 3 we plot the two body KK parity violating decay width of $B_{1}$ as a function of $h$ with $R^{-1}$ as a free parameter. The band corresponds to the variation of $R^{-1}$ : the top line for $R^{-1}=1 \mathrm{TeV}$ and the bottom line for $R^{-1}=300 \mathrm{GeV}$. $h$ is varied between $10^{-10}$ to 0.01 . It appears that if $h \sim 10^{-6}, B_{1}$ decay will lead to a secondary vertex, while if $h \sim 10^{-8}$, the path length is of the order of a metre and it can decay outside the detector. In this case, the signals will be identical to that of mUED but the LKP may be considerably heavier than the overclosure limit of $\sim 1 \mathrm{TeV}$.

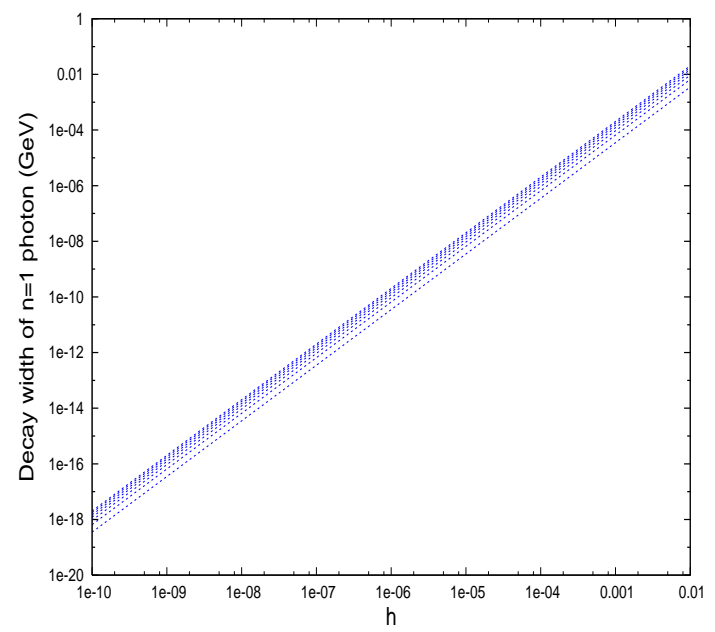

Figure 3: The decay width of $B_{1}$ as a function of $h$ for different values of $R^{-1}$, from $1 \mathrm{TeV}$ (top of the band) to $300 \mathrm{GeV}$ (bottom).

Case $2\left(l_{1}\right.$ LKP): At the $n=1$ levels, there are two leptons, $l_{1}$ and $l_{2}$. For small values of $h, l_{1}$ is dominantly singlet and $l_{2}$ is dominantly doublet. Thus, $l_{1}$ decays almost entirely to $l+\gamma$ or $l+Z$; the $\nu+W$ channel will be negligible due to the chiral nature of $l_{1}$. 
If $l_{1}$ is the NLKP, it will decay with almost $100 \%$ branching ratio to $l+B_{1}$ if $h$ is small and $l_{1}-B_{1}$ splitting is at least $1 \mathrm{GeV}$. If $h$ is large, the KK parity violating decay modes will start competing with the KK conserving ones, since the latter becomes more phase space suppressed with increasing $h$, while the former channel gets enhanced. Here we show the KK parity conserving and KK parity violating branching ratios of the singlet lepton for three benchmark values of $h$. In the first case $l_{1}$ is above the $B_{1}$ so that KK conserving decay width is not suppressed. In the third case $M_{B_{1}}>M_{l_{1}}$ so that all KK conserving channels are closed. In this case it can decay dominantly to SM $Z$ boson. The second case corresponds to the minimal UED where $h=0$.

\begin{tabular}{||c|c|c|c|c||}
\hline$R^{-1}$ & $h$ & $\operatorname{Br}\left(l_{1} \rightarrow B_{1}\right)$ & $\operatorname{Br}\left(l_{1} \rightarrow \gamma\right)$ & $\operatorname{Br}\left(l_{1} \rightarrow Z\right)$ \\
\hline 500 & -0.01 & 74.9 & 19.6 & 5.5 \\
\hline 500 & 0.0 & 100 & 0 & 0 \\
\hline 500 & 0.01 & 0 & 77.9 & 22.1 \\
\hline
\end{tabular}

Table 2: Branching fractions of $l_{1}$.

We also show the decay channels and branching fractions of $l_{2}$, which can at most be an NLKP candidate (it can never go below $l_{1}$ for small and uniform values of $h$ ). Note that the KK parity violating channels may become important for large $h$. However, neither $l_{1}$ nor $l_{2}$ can decay outside the detector; this happens only for very tiny values of $h$ where $B_{1}$ is the LKP.

\begin{tabular}{||c|c|c|c|c|c||}
\hline$R^{-1}$ & $\mathrm{~h}$ & $\operatorname{Br}\left(l_{2} \rightarrow B_{1}\right)$ & $\operatorname{Br}\left(l_{2} \rightarrow \gamma\right)$ & $\operatorname{Br}\left(l_{2} \rightarrow Z\right)$ & $\operatorname{Br}\left(l_{2} \rightarrow W\right)$ \\
\hline 500 & -0.01 & 50.6 & 14.3 & 5.5 & 29.6 \\
\hline 500 & 0.0 & 100 & 0 & 0 & 0 \\
\hline 500 & 0.01 & 17.9 & 23.8 & 9.1 & 49.1 \\
\hline
\end{tabular}

Table 3: Branching fractions of $l_{2}$.

Case $3\left(H_{1}^{ \pm}\right.$LKP): The third possibility, which only occurs for heavy SM Higgs, is the charged Higgs LKP. The KK parity violating decays of $H_{1}^{ \pm}$and other excited scalars occur through the admixture of the $n=1$ state with the physical lowest-lying state, as there is no such parity violating term for the Yukawa sector to start with. $H_{1}^{ \pm}$can also decay to $f \bar{f} V_{0}$, where $V_{0}$ is a SM gauge boson. This proceeds through the virtual $n=1$ gauge boson state. The exact branching fractions depend on $h$; for very small $h, H_{1}^{ \pm}$can decay outside the detector and one observes the thick charged track, something reminiscent of a long-lived chargino.

\section{Collider Phenomenology}

The characteristic collider signal of the mUED model is SM particles with low transverse energy and a huge amount of missing energy, which is very similar to the R-parity conserving SUSY models. Missing energy comes from the stable neutral LKP, $B_{1}$, which does not interact with the detector. But when KK parity is broken we lose the missing energy part of the signals because LKP is no longer stable. The only source of missing energy in this case is the SM neutrino which may come from the decay of $B_{1}, W_{1}$ and $Z_{1}$ (see fig. (4). In our analysis we consider only those cases where $h$ is so small that single production of KK excitations is not allowed at colliders, but the strength is sufficient to allow KK particles to decay within the detector. In other words, we do not consider any single production but study the effect of KK parity violation at the last stage of the cascade where LKP is produced. The model follows the mUED allowed productions and decays except the LKP decay. Also for small value of $h$, the mass spectum remains almost unchanged.

We are now in a position to discuss qualitatively the experimental signatures of such a model. At the LHC, KK excitations can be produced mainly through strong interaction. The dominant processes are the pair production of $n=1$ colored objects:

$$
p p \longrightarrow g_{n=1} q_{1} / q_{2}, \quad g_{n=1} g_{n=1}, \quad q_{1} / q_{2} q_{1} / q_{2},
$$


where $g_{n=1}$ is the $n=1$ gluon and $q_{1} / q_{2}$ are the dominantly singlet or doublet $n=1$ quark states. The production processes of electroweak strength are

$$
p p \longrightarrow W W, W Z, Z Z, \quad l_{1} \bar{l}_{1}, \quad l_{2} \bar{l}_{2}, \quad \nu_{2} \bar{\nu}_{2}
$$

where all gauge bosons are the $n=1$ states. The cascade decays of the produced excited particles result in final state with two $B_{1}$. In the mUED case, $B_{1}$ is stable and thus escapes the detector. The singlet quark can decay only to $B_{1}$, whereas doublet quarks can decay mostly to $W_{1}$ or $Z_{1}$. The hadronic decay modes of $W_{1}$ and $Z_{1}$ are closed, and they decay universally to all lepton flavours. The leptons finally decay to $B_{1}$. Thus, the final state signature of mUED is $n$ jets $+m$ leptons + missing $E_{T}$.

When KK parity is broken, $B_{1}$ can decay two SM fermions. Some of the possible decay chains are shown in figure 4. We get a huge number of different final states depending on the deacy pattern of $B_{1}$. The decay of the LKP will increase the particle multiplicity in the final state and we expect an excess in the SM particles. This is similar to the R-parity violating SUSY scenarios. The decay pattern of $B_{1}$ shows that it can decay invisibly (although the branching is small $\sim 8 \%$ ). This gives rise to missing energy in the final state. The SM neutrinos may also come from $W_{1}$ or $Z_{1}$ decay.
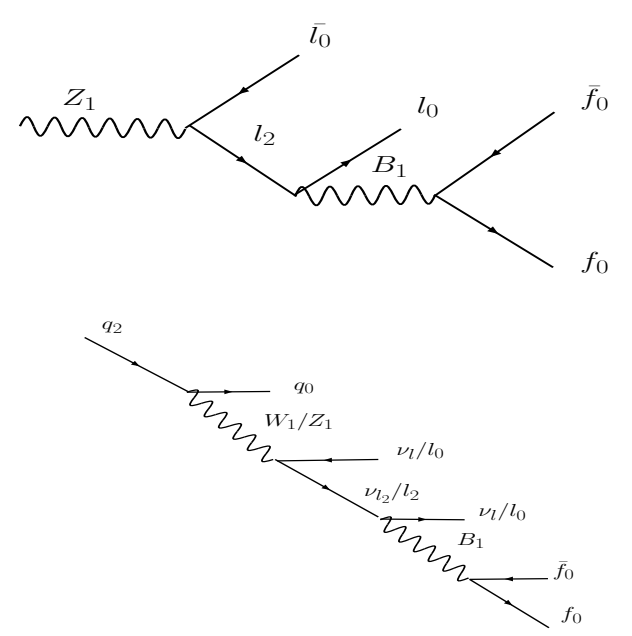
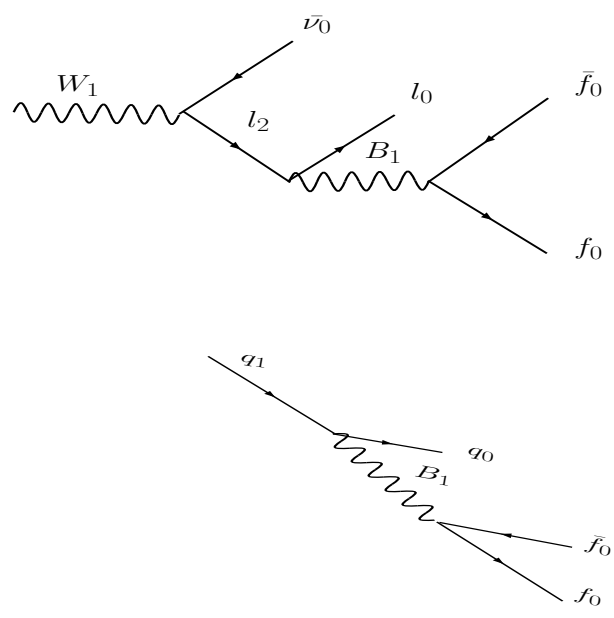

Figure 4: Feynman diagrams of $q_{1}, \tilde{q_{1}}, W_{1}$ and $Z_{1}$ decay

In the mUED model the spectrum is highly degenerate even after radiative correction. So the missing $E_{T}$ is not large (the distribution has a peak at about 100 to $150 \mathrm{GeV}$ ). The signals with no missing energy are not easy to detect, because of the huge SM backgrounds. We will get 4 lepton or 2 lepton plus missing energy or 2 leptons plus 2 quarks or two quarks plus missing energy, or 2 lepton plus 2 quarks from $Z_{1}$ decay. $Z_{1}$ can also decay invisibly, although the branching ratio is small. The $W_{1}$ decays are very similar to the $Z_{1}$ decay. The final state coming from $g_{1} g_{1}$ must contain $N \geq 4$ jets. It may contain a large number of leptons $(\leq 8)$ too, without or with missing energy. If $B_{1}$ decays to $t \bar{t}$ the final states will turn out to be very complicated. If we consider the production processes like $g_{1} q_{1}$ or $q_{1} q_{1}$, final state will again be multijet ( $\geq 3$ or 2 ) and multilepton without or with missing energy. This new feature of the UED model may be difficult to extract from the usual multijet plus multilepton signal of the SM, because of the huge SM background. Separation of signal from backgrounds is nontrivial.

On the other hand, hadronically quiet multilepton signals (may or may not be accompanied by missing energy) are more interesting, though the initial production cross-section is suppressed by the ratio $\alpha / \alpha_{s}$. This signal may come from the electroweak production processes noted earlier. Let us look at the signal $n$ leptons plus missing energy, where $4 \leq n \leq 7$. For $R^{-1}=500 \mathrm{GeV}$, the branching fractions are as follows: 


\begin{tabular}{|c|c|c|c|}
\hline No & Processes & final states & $\begin{array}{c}\text { Branching } \\
\text { (In percent) }\end{array}$ \\
\hline 1 & $\overline{W_{1} W_{1}}$ & $\begin{array}{l}61+p t \\
41+p t\end{array}$ & $\begin{array}{c}15.1 \\
3.0\end{array}$ \\
\hline 2 & $Z_{1} Z_{1}$ & $\begin{array}{l}61+p t \\
41+p t\end{array}$ & $\begin{array}{c}9.0 \\
12.4\end{array}$ \\
\hline 3 & $Z_{1} B_{1}$ & $41+p_{T}$ & 21.2 \\
\hline 4 & $W_{1} B_{1}$ & $51+p_{7}$ & 27.1 \\
\hline 5 & $W_{1} Z_{1}$ & $\begin{array}{l}51+p_{t} \\
71+p_{t}\end{array}$ & $\begin{array}{l}21.2 \\
15.1\end{array}$ \\
\hline 6 & $l_{1} L_{1}$ & $4 \mathrm{l}+p_{t}$ & 6.1 \\
\hline 7 & $l_{1} L_{1}$ & $41+p_{t}$ & 6.1 \\
\hline 8 & $n_{1} N_{1}$ & $41+p t$ & 15.1 \\
\hline
\end{tabular}

Table 4: Branching fractions for multilepton final states.

We plot the variation of 4-7 leptons + missing energy cross section as a function of $R^{-1}$ ( $h$ does not play any role here except forcing the decay of the LKP). The numerical cumputations were done with the CalcHEP package [17, augmented by the implementation of UED. The branching fractions, multiplied by the respective production cross-sections, give the final signal cross-section and hence the event rate. It can be seen from figure 5 that the $6 \ell+p \nmid$ signal has the highest cross-section, closely followed by that of $4 \ell+p t$.

The SM backgrounds are under comparative control. First, same-flavor unlike-sign dilepton invariant mass veto at $M_{Z}$ removes the most important background ( $W W Z \rightarrow 4 \ell+p t$ is about $86 \mathrm{fb}$ at the LHC). While the $W$-backgrounds, associated with neutrinos, cannot be removed in this way, they are further suppressed by higher powers of $\alpha$. What may be problematic is to detect all the leptons coming from the excited states. Some of them can be very soft, coming from decay between closely spaced levels, which will probably missed by the acceptance of the detector. However, they come from the KK-conserving decays at the first stage of the cascade. The KKPV decays produce hard leptons, which should be easily detectable. In short, one should be able to discriminate such a scenario from other competing new physics scenarios, as well as from the SM itself, from the event rate and topology of the multilepton final state. A detailed study is outside the scope of this paper and will be taken up later.
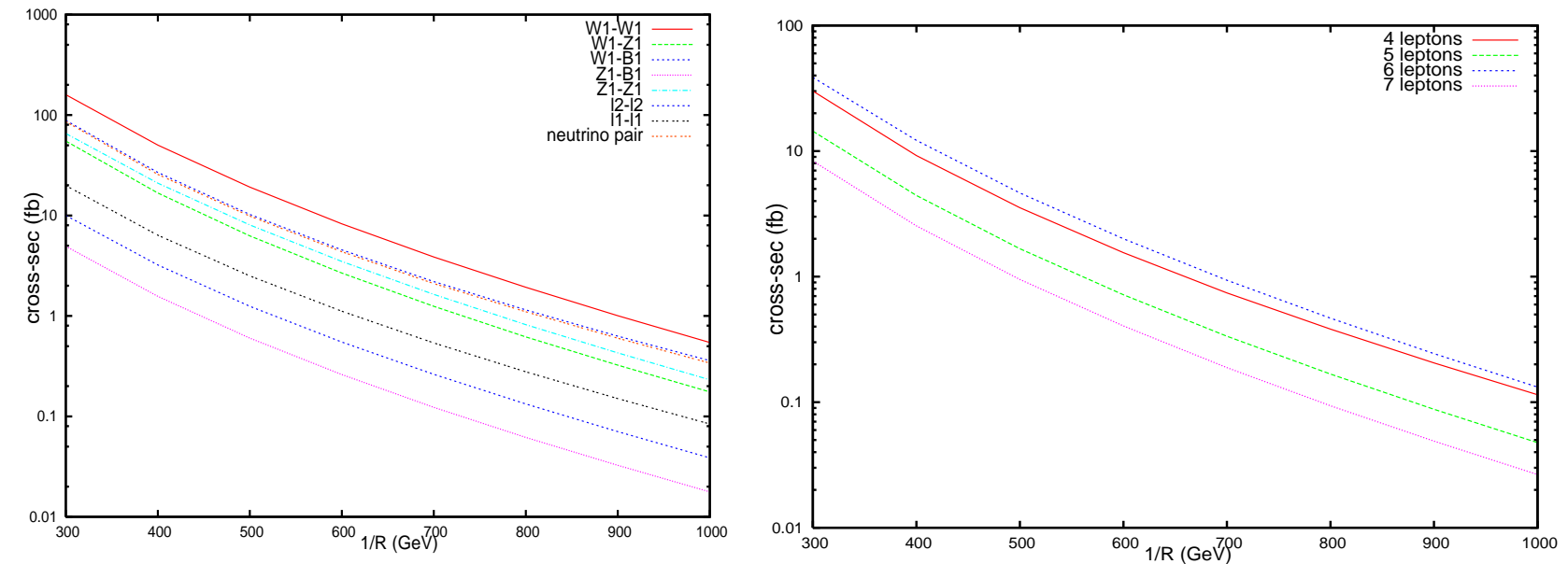

Figure 5: Left panel: cross-section for electroweak production at the LHC. Right panel: cross-section for multilepton plus missing energy signal. 


\section{Summary}

In this paper we have discussed the phenomenology of KK parity violation in the UED model through an asymmetrically fixed-point located term. This term removes the $\mathcal{Z}_{2}$ parity of $y \rightarrow y+\pi R$ and hence KK parity is violated. Thus, the lightest $n=1$ particle, the LKP, is no longer stable and a cold dark matter candidate. This removes the overclosure bound and answers the question posed in the Introduction, viz., whether non-observation of UED signals at the LHC will invalidate this model, in the negative. This also allows the introduction of gravitons in the model even for comparatively low values of $R^{-1}$.

The model that we have discussed is rather simplistic. The KKPV strength may be different for different flavors, and that may give rise to interesting flavor-changing constraints, but we have assumed the same $h$ for all fermions. We have also kept the coupling small so that the field expansion is valid and there is no single production of the excited states at the colliders.

The removal of dark matter LKP opens up various possibilities in the LKP-NLKP phase diagram, which we have studied. This, in turn, corresponds to different types of signals in the colliders. Depending on the strength of the coupling the LKP may decay inside or outside the detector. If it decays inside the detector, the multilepton final states should be useful to prove the validity of this model. For large values of the SM Higgs boson mass and a small KK-parity violating coupling $h \sim 10^{-8}, H_{1}^{ \pm}$, which becomes a long-lived LKP to decay outside the detector, leaves its characteristic charged track.

\section{Acknowledgements}

The author thanks Anirban Kundu for many useful discussions. He is supported by a research fellowship of UGC, Govt. of India, and also acknowledges the Regional Centre for Accelerator-based Particle Physics (ReCAPP), HRI, Allahabad, for a young associateship.

\section{References}

[1] T. Appelquist, H.C. Cheng, and B.A. Dobrescu, Phys. Rev. D 64, 035002 (2001).

[2] H.C. Cheng, K.T. Matchev, and M. Schmaltz, Phys. Rev. D 66, 036005 (2002).

[3] J. A. R. Cembranos, J. L. Feng and L. E. Strigari, Phys. Rev. D 75, 036004 ( 2007).

[4] G. Servant and T.M.P. Tait, Nucl. Phys. B650, 391 (2003);

M. Kakizaki et al., Nucl. Phys. B735, 84 (2006);

D. Hooper and S. Profumo, Phys. Rept. 453, 29 (2007).

[5] H.C. Cheng, K.T. Matchev, and M. Schmaltz, Phys. Rev. D 66, 056006 (2002).

[6] I. Antoniadis, Phys. Lett. B246, 377 (1990);

C. Macesanu, C.D. McMullen, and S. Nandi, Phys. Rev. D 66, 015009 (2002);

C. Macesanu, C.D. McMullen, and S. Nandi, Phys. Lett. B546, 253 (2002);

H.C. Cheng, Int. J. Mod. Phys. A18, 2779 (2003);

A. Muck, A. Pilaftsis, and R. Rückl, Nucl. Phys. B687, 55 (2004);

A. Datta, K. Kong, and K.T. Matchev, Phys. Rev. D 72, 096006 (2005);

C. Macesanu, Int. J. Mod. Phys. A 21, 2259 (2006).

[7] G. Bhattacharyya et al., Phys. Lett. B628, 141 (2005);

M. Battaglia et al., J. High Energy Physics 0507, 033 (2005).

[8] T.G. Rizzo, Phys. Rev. D 64, 095010 (2001).

[9] B. Bhattacherjee and A. Kundu, Phys. Lett. B627, 137 (2005);

B. Bhattacherjee, arXiv:hep-ph/0608227.

A. Datta and S. K. Rai, Int. J. Mod. Phys. A23, 519 (2008). 
S. K. Rai, Int. J. Mod. Phys. A23, 82 (2008);

B. Bhattacherjee, A. Kundu, S. K. Rai and S. Raychaudhuri, arXiv:0805.3619 [hep-ph].

[10] F. del Aguila, M. Perez-Victoria and J. Santiago, J. High Energy Physics 0302, 051 ( 2003);

F. del Aguila, M. Perez-Victoria and J. Santiago, Acta Phys. Polon. B 34, 5511 (2003)

[11] C. D. McMullen and S. Nandi, J. Phys. G 35, 095002 ( 2008).

[12] R. N. Mohapatra, S. Nussinov and A. Perez-Lorenzana, Phys. Rev. D 68, 116001 (2003)

[13] S. Matsumoto and M. Senami, Phys. Lett. B633, 671 (2006);

M. Kakizaki, S. Matsumoto and M. Senami, Phys. Rev. D 74, 023504 (2006).

[14] H. Georgi, A.K. Grant, and G. Hailu, Phys. Lett. B506, 207 (2001).

[15] M. Puchwein and Z. Kunszt, Annals Phys. 311, 288 (2004).

[16] B. Bhattacherjee and A. Kundu, J. Phys. G 32, 2123 (2006);

B. Bhattacherjee and A. Kundu, Phys. Lett. B653, 300 (2007).

[17] A. Pukhov, hep-ph/0412191, and http://www.ifh.de/p̃ukhov/calchep.html. 